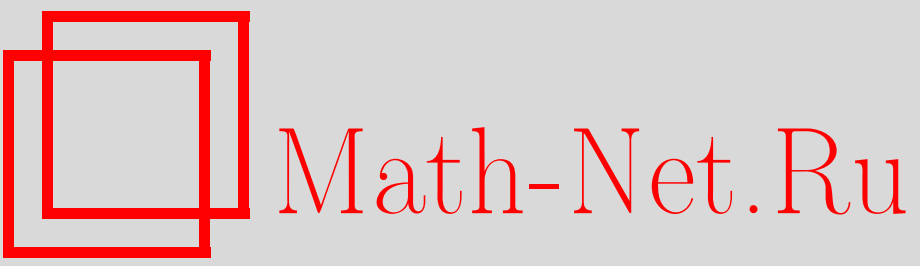

Л. О. Чехов, Интегрируемые деформации систем на графах с петлями, УМН, 2002, том 57, выпуск 3, 169-170

DOI: https://doi.org/10.4213/rm514

Использование Общероссийского математического портала Math-Net.Ru подразумевает, что вы прочитали и согласны с пользовательским соглашением

http://www.mathnet.ru/rus/agreement

Параметры загрузки:

IP : 18.234 .197 .8

26 апреля 2023 г., 05:21:34 


\title{
ИНТЕГРИРУЕМЫЕ ДЕФОРМАЦИИ СИСТЕМ НА ГРАФАХ С ПЕТЛЯМИ
}

\author{
Л. О. ЧЕХОВ
}

Изучение спектральных свойств и процессов рассеяния для операторов шредингеровского типа на бесконечных деревьях [1], [2] привело к обнаружению ряда неожиданных результатов, в том числе связи между матрицей рассеяния ( $S$-матрицей $)$ для бесконечного графа типа дерева с циклами и $L$-функцией Артина-Сельберга [3]. Тем не менее, не удалось найти никакой подходящей интегрируемой деформации операторов второго порядка, которая бы была аналогична соответствующей деформации для решеток $\mathbb{Z}\left((L, A)\right.$-пары типа цепочки Тоды) и $\mathbb{Z}_{2}((L, A, B)$-тройки) (см. [4]).

Деформация произволнного симметрического оператора четвертого порядка $L$ на граф̆е $T_{3}$ (бесконечное однородное дерево с вершинами валентности три) в форме $(L, A, B)$-тройки, $\dot{L}=L A-B L$, была построена Кричевером и Новиковым [5]. Эта деформация сохраняет спектральные данные нулевого уровня энергии $L \psi=0$. В работе [6] интегрируемые системы Кричевера-Новикова были обобщены на случай произвольного (неоднородного) графа типа дерева $T$.

Произвольный граф Г с конечным числом циклов представим единственным (с точностью до изоморфизмов) образом как фактор универсального накрьвающего дерева $T$ относительно действия конечнопорожденной подгруппы $\Delta$ полной группы симметрий графа типа дерева $T$, $\Gamma=T / \Delta$. Рассматриваются операторы $L$, действующие на пространстве функций $\psi_{P}$, заданных в вершинах графа $\Gamma$. Расстояние $d\left(X_{1}, X_{2}\right) \in \mathbb{Z}_{+} \cup\{0\}$ на древесном графе измеряется числом ребер, содержащихся в единственном пути, соединяющем вершины $X_{1}$ и $X_{2}$. Порядком уравнения $L \psi=0$, где $(L \psi)_{P}=\sum_{X} b_{P, X} \psi_{X}$, назьвается максимальный диаметр $\max _{P} d\left(X_{1}, X_{2}\right)$ : $b_{P, X_{1}} \neq 0, b_{P, X_{2}} \neq 0$ или $b_{X_{1}, X_{2}} \neq 0$. В общем графе существует множество путей, соединяюших вершины $X_{1}$ и $X_{2}$. В дальнейшем будет предполагаться, что теория рассматривается на накрьвающем дереве $T$ с наложенным условием периодичности всех функций $\psi_{X}$ и коэффициентов операторов $L, A$, и $B$ по отношению к действию подгруппы $\Delta$ группы симметрии. На факторизованном графе $\Gamma$ это означает, что коэффициенты операторов ассоциированы с путяли соответствующей длины, связывающими прообразы $\widetilde{X}_{1}, \widetilde{X}_{2} \in T$ соответствующих вершин $X_{1}, X_{2} \in \Gamma$. Рассмотрим сначала произвольный граф̆ без петель.

Теорема 1 [6]. Вещественный самосопряженный оператор L четвертого порядка на графе $T$, не содержащем петель, допускает изоспектральную деформацию нулевого уровня энергии $L \psi=0$, имеющую вид $(L, A, B)$-тройки $\left[B=A^{t}\right]$,

$$
\dot{L}=L A+A^{t} L,
$$

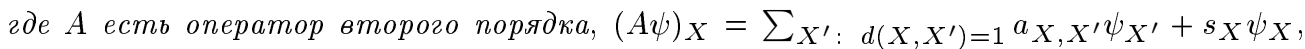
тогда и только тогда, когда оператор $L$,

$$
(L \psi)_{X}=\sum_{X^{\prime \prime}: d\left(X, X^{\prime \prime}\right)=2} b_{X, X^{\prime \prime}} \psi_{X^{\prime \prime}}+\sum_{X^{\prime}: d\left(X, X^{\prime}\right)=1} r_{X, X^{\prime}} \psi_{X^{\prime}}+\rho_{X} \psi_{X}
$$

(такой, что все $b_{X, X^{\prime \prime}}>0$ ), можсет бить представлен в виде

$$
L=Q^{t} Q+u_{X}
$$

где $Q$ есть оператор второго порядка, $(Q \psi)_{X}=\sum_{X^{\prime}: d\left(X, X^{\prime}\right)=1} q_{X, X^{\prime}} \psi_{X^{\prime}}+v_{X} \psi_{X}$.

В случае, когда $b_{X, X^{\prime \prime}} \equiv b_{X^{\prime \prime}, X}>0$, коэффициенты $q_{X, X^{\prime}}$ однозначно определяются по коэффициентам $b_{X, X^{\prime \prime}}$, и наоборот. В частности, для любых трех вершин $X_{1}, X_{2}, X_{3}$, таких, что $X_{1} \neq X_{3}$ и $d\left(X_{i}, X_{i+1}\right)=1, i=1,2, b_{X_{1}, X_{3}}=q_{X_{1}, X_{2}} q_{X_{3}, X_{2}}$. (Произвольньй оператор

Работа выполнена при поддержке Российского фонда фундаментальных исследований (грант № 01-01-00549) и фонда INTAS (грант № 99-1782). 
четвертого порядка $L$ на дереве $T_{3}$ допускает представление (3), а потому и изоспектралшную деформацию (1) [5].)

Коэффициенты $a_{X, X^{\prime}}$ оператора деформации $A$ однозначно вьражаются (с точностью до глобальной мультипликативной константы) через $q_{X}, Y$. Для произвольных трех вершин $X_{1}, X_{2}$, $X_{3}: d\left(X_{i}, X_{i+1}\right)=1, i=1,2$, получим ${ }^{1}$

$$
\frac{a_{X_{2}, X_{1}}}{q_{X_{1}, X_{2}}}=\frac{a_{X_{2}, X_{3}}}{q_{X_{3}, X_{2}}} \text { и } a_{X_{3}, X_{2}}=-a_{X_{2}, X_{1}} \frac{q_{X_{2}, X_{3}}^{2}}{q_{X_{1}, X_{2}} q_{X_{3}, X_{2}}}
$$

(в частности, при $X_{1}=X_{3}$ получим $a_{X_{1}, X_{2}}=-a_{X_{2}, X_{1}} q_{X_{2}, X_{1}}^{2} / q_{X_{1}, X_{2}}^{2}$ ).

Обозначим через $\left\{X_{j}^{\prime}\right\}$ и $\left\{X_{i}^{\prime \prime}\right\}$ множества вершин, соседних по отношению к соответствующим вершинам $X_{1}$ и $X_{2}$ и таких, которые не совпадают ни с $X_{1}$, ни с $X_{2}$. Явные формулы для деформации имеют вид:

$$
\begin{aligned}
\dot{b}_{X_{1}, X_{3}}= & r_{X_{1}, X_{2}} a_{X_{2}, X_{3}}+a_{X_{2}, X_{1}} r_{X_{2}, X_{3}}+b_{X_{1}, X_{3}}\left(s_{X_{1}}+s_{X_{3}}\right), \\
\dot{r}_{X_{1}, X_{2}}= & \sum_{X^{\prime \prime}} b_{X_{1}, X^{\prime \prime}} a_{X^{\prime \prime}, X_{2}} \\
& +\sum_{X^{\prime}} b_{X_{2}, X^{\prime}} a_{X^{\prime}, X_{1}}+\rho_{X_{1}} a_{X_{1}, X_{2}}+\rho_{X_{2}} a_{X_{2}, X_{1}}+r_{X_{1}, X_{2}}\left(s_{X_{1}}+s_{X_{2}}\right), \\
\dot{\rho}_{X_{1}}= & \sum_{\left\{X^{\prime}\right\} \cup X_{2}} r_{X_{1}, X^{\prime}}\left(a_{X^{\prime}, X_{1}}+a_{X_{1}, X^{\prime}}\right)+2 \rho_{X_{1}} s_{X_{1}} .
\end{aligned}
$$

Лемма 1 [6]. Для произвольного графа типа дерева деформация (5) с учетом формул (4) сохраняет условие факторизации (3).

ТЕОРемА 2. Для произвольного графа $\Gamma_{3}$ с вериинами валентности три и произвольным конечным числом петель деформация (5) сохраняет свойство периодичности тогда и только тогда, когда для каждого замкнутого иикла с последовательно пронумерованными (ичклически упорядоченными) вершинами $\left\{X_{i}\right\}_{i=1}^{n}, X_{n+1} \equiv X_{1}$, выполнены следующие два условия:

$$
\begin{aligned}
& \text { (a) } \prod_{k=1}^{n}(-) \frac{q_{X_{k}, X_{k+1}}}{q_{X_{k+1}, X_{k}}}=1 \quad \text { (условие кочикла, см. [5]), } \\
& \text { (b) } \sum_{i=1}^{n} r_{X_{i}, X_{i+1}} \prod_{k=1}^{i-1}(-) \frac{q_{X_{k}, X_{k+1}}}{q_{X_{k+2}, X_{k+1}}}=0 .
\end{aligned}
$$

ЗАмечАниЕ. Доказательство теоремы 2 представляет собой достаточно длинное, но прямое вычисление с использованием формул (4) и (5). Заметим, что производная по времени от условия (a) дает удвоенное условие (b), и надо лишь показать, что производная по времени от условия (b) тождественно равна нулю, если выполнены оба условия (a) и (b). Посколшку как усло-

вие (a), так и условие (b) удовлетворяют свойству коцикла, достаточно наложить эти условия только на базисные циклы графра.

\section{СПИСОК ЛИТЕРАТУРЫ}

[1] С. П. Новиков // УМН. 1997. Т. 52. №6. С. 177-178. [2] S. P. Novikov // Fields Inst. Commun. 1999. V. 24. Р. 397-413. [3] Л.О. Чехов // УМН. 1999. Т. 54. №6. С. 109-148. [4] С. П. Новиков, И. А. Дынников // УМН. 1997. Т. 52. № 5. С. 175-234. [5] И. М. Кричевер, С. П. Новиков // УМН. 1999. Т. 54. №6. С. 149-150. [6] Л. О. Чехов, Н. В. Пузырникова // УМН. 2000. Т. 55. № 5. С. 181-182.

Математический институт им. В. А. Стеклова РАН

Принято редколлегией E-mail: chekhov@mi.ras.ru

\footnotetext{
${ }^{1}$ В этой формуле в работе [6] содержалась опечатка.
} 